\title{
«Islam de l'extérieur, musulmans de l'intérieur » : deux visions après le 11 septembre 2001
}

"External Islam, Domestic Muslims ", two Perspectives after September 11th 2001

Jocelyne Cesari

\section{(2) OpenEdition \\ Journals}

Édition électronique

URL : http://journals.openedition.org/conflits/738

DOI : $10.4000 /$ conflits.738

ISSN : $1777-5345$

Éditeur :

CCLS - Centre d'études sur les conflits lilberté et sécurité, L'Harmattan

Édition imprimée

Date de publication : 1 décembre 2001

Pagination : $97-115$

ISBN : 2-7475-2227-X

ISSN : $1157-996 X$

Référence électronique

Jocelyne Cesari, « «Islam de l'extérieur, musulmans de l'intérieur » : deux visions après le 11

septembre 2001 », Cultures \& Conflits [En ligne], 44 | hiver 2001, mis en ligne le 22 mars 2006, consulté le 30 mars 2021. URL : http://journals.openedition.org/conflits/738 ; DOI : https://doi.org/10.4000/

conflits.738

Ce document a été généré automatiquement le 30 mars 2021.

Creative Commons License 


\title{
«Islam de l'extérieur, musulmans de l'intérieur »: deux visions après le 11 septembre 2001
}

\author{
"External Islam, Domestic Muslims", two Perspectives after September 11th \\ 2001
}

Jocelyne Cesari

Il est toujours surprenant de constater à quel point la perception dominante de l'islam l'érige en « inquiétante étrangeté » comme si entre « eux et nous » il n'y avait aucune valeur partagée alors que l'islam n'est jamais que la troisième branche du tronc monothéiste. Les valeurs de l'islam sont donc dans l'absolu, comparables à celles des autres religions monothéistes et pourtant surgit régulièrement la vision d'une religion " orientale» radicalement opposée et combative vis-à-vis de nos valeurs "judéochrétiennes $»^{1}$. Il faut en chercher les raisons dans une histoire faite de confrontations entre l'Europe et le monde musulman dont l'espace méditerranéen a été le décor principal depuis l'époque médiévale. Ce que nous croyons savoir de l'islam est en grande partie le produit d'une vision bâtie sur des siècles d'opposition tout autant politique que religieuse. La réalité mouvante et paradoxale des musulmans de l'intérieur comme de l'extérieur, du comportement le plus privé jusqu'aux aspects les plus collectifs, disparait alors sous le poids de représentations sédimentées au fil des siècles. Ces représentations s'agrègent à partir de moments historiques et de rencontres qui vont cristalliser de manière durable des tryptiques d'images différentes et parfois contradictoires telles que «violence, hérésie et débauche» ou encore " sensualité, brutalité, cruauté »².

Depuis l'ouvrage d'Edward Said, L'orientalisme. L'Orient créé par l'Occident ${ }^{3}$, la corrélation entre l'impérialisme et la tradition scientifique orientaliste n'est plus à faire. En effet, cette discipline allait s'affirmer au cours du XIXe et du suivant, à la fois comme une tradition savante, un mode de pensée fondé sur la distinction ontologique entre Orient et Occident et comme une institution au service de la domination. De Sylvestre de Sacy à Louis Massignon en passant par Ernest Renan, cette théorie et cette praxis a consisté à 
reformuler dans le langage scientifique de l'époque, l'ensemble des structures héritées du passé en contribuant à pérenniser une vision essentialiste et totalisante de l'islam. L'Orient et avec lui l'islam apparaissent comme une « nécessité intellectuelle ». En effet, le criticisme historique et le moralisme scientifique de la philologie, permettaient à la critique rationaliste de s'affirmer dans l'étude des religions et de légitimer une opposition entre d'un côté, les religions et en particulier l'islam et de l'autre, la science. A n'en pas douter, bon nombre de nos contemporains, acquiesceraient aux termes utilisés par Ernest Renan pour exprimer cette opposition :

«Ce qui distingue en effet le musulman, c'est la haine de la science, c'est la persuasion que la recherche est inutile, frivole, presque impie, la science de la nature parce qu'elle est une concurrence faite à Dieu, la science historique parce que s'appliquant à des temps antérieurs à l'islam, elle pourrait raviver d'anciennes erreurs $»^{4}$.

Sans remettre en cause les apports sur la langue ou la culture de l'orientalisme, ni négliger les profondes transformations apportées par les sciences humaines dans l'étude du monde arabo-musulman, il faut cependant être attentif aux présupposés qui ont longtemps sous-tendu tout discours y compris à prétention scientifique sur l'islam et les musulmans parce qu'ils s'inscrivaient dans une vison inégalitaire entre cultures, allant de pair avec la geste coloniale. Et si l'orientalisme manifeste a été profondément renouvelé par la sociologie, l'anthropologie ou la science politique, il n'en demeure pas moins que l'orientalisme latent, somme des représentations accumulées, continue d'agir. De ce point de vue, Edward Saïd a raison d'affirmer que l'Orient et l'islam n'existent pas: ils sont des topos, des ensembles de références, une somme de caractéristiques liées à un imaginaire.

Dans cette vision, nourrie par des justifications scripturaires, l'islam est toujours présenté comme un système clos, "un prototype des sociétés traditionnelles fermées ", ce qui dénie, toute capacité de transformations aux hommes et aux sociétés d'islam. De telles perceptions ont bien évidemment une portée idéologique qui depuis le XIXe siècle légitiment toutes les entreprises de domination sur ces parties du monde.

Or, cette approche essentialiste dépeinte et critiquée par E. Saïd est loin d'avoir disparu. Il est frappant de constater que les considérations sur l'islam comme facteur de trouble dans les relations internationales depuis les années 1980 sont légitimées par des représentations sédimentées sur plusieurs siècles qui sembleraient très familières à l'honnête homme du XVIIIe siècle. Les même attributs réifiants sont activés et recomposés en fonction d'enjeux internationaux et domestiques nouveaux. Il semble que les attentats du 11 septembre 2001 ont renforcé cette vision sécuritaire et ont en même temps créé, pour la première fois, les conditions pour une dissociation entre le musulman de l'intérieur et l'ennemi politique.

La menace islamique ou la logique de guerre ${ }^{5}$

L'islam politique est devenu l'élément dominant pour appréhender et comprendre les sociétés musulmanes et ce, bien avant septembre 2001. En l'espace de deux décades, les mouvements politiques utilisant les références islamiques comme moyens de contestation politique ont concentré sur eux l'attention non seulement des classes politiques du monde musulman mais aussi des media et des universitaires en Europe et aux Etats-Unis. Certes leur force politique et l'usage de la violence dont certains font preuve, sont les raisons d'un tel intérêt. Mais il en résulte une vision unique et simplifiée qui de Téhéran à Alger en passant par Kaboul donne à voir une seule dimension sémantique qui jouant des confusions des images comme des termes, 
réactualise la constellation de représentations déjà évoquées autour de la violence et du fanatisme. Cette perception ne laisse plus de place à d'autres aspects du monde musulman et le citoyen qui s'en tient à son journal télévisé est bien en peine de comprendre ce qui se joue aujourd'hui en Algérie, en Egypte, en Iran ou en Afghanistan et ne peut que s'effrayer $d u$ phénomène islamique tant tous les registres d'appartenance et de mobilisation au nom de l'islam ne sont jamais distingués. Comment s'étonner alors que ces mêmes citoyens lorsqu'ils sont interrogés, par exemple aux Etats-Unis en novembre 1994, considèrent à $61 \%$ que la résurgence islamique est un danger? Ce phénomène étant pour eux, le plus souvent synonyme de terrorisme international. En Europe, la peur s'exprime de manière identique : en 1991, $51 \%$ des Français interrogés pensaient que le principal danger pour la France provenait du Sud. L'Irak, l'Iran, la Libye et l'Algérie étant cités comme les quatre pays les plus craints.

Il est vrai qu'une série d'événements « explosifs » ont durant les vingt dernières années fourni les images dominantes de cet islam militant : la révolution iranienne et la prise d'otages de l'ambassade américaine, l'assassinat d'Anwar El Sadate, les otages au Liban, l'affaire Rushdie, la crise algérienne, le conflit en Afghanistan. L'islam désormais perçu comme une menace dans les relations internationales, est en passe de prendre la place $\mathrm{du}$ communisme dans les principaux facteurs de risque. Dans les nomenclatures de l'OTAN comme dans celles qui prévalent au Pentagone, un certain nombre de pays ou de région « à caractère islamique » figurent en bonne place qu'il s'agisse de l'Iran ou du Soudan « labellisés » terroristes par l'administration américaine.

Cette vision stratégique prend appui sur un certain nombre d'écrits à caractère scientifique suscités par la fin du clivage Est/Ouest. Dans un article paru dans International Affairs en juillet 1991, Barry Buzan a été l'un des premiers à tirer les conséquences de la disparition de l'ennemi communiste: cette situation nouvelle ne peut que modifier les rapports de force au centre et précipiter «la collision des identités culturelles ». Dans cette perspective, les rapports entre islam et Occident sous forme de guerre sociale froide ne peut que favoriser la consolidation de l'identité européenne à un moment crucial de son unification. Mais c'est surtout l'approche de Samuel Huntington à travers un article de Foreign Affairs en 1993 puis d'un livre paru en 1996, qui a connu le plus large écho, à tel point que «le clash des civilisations » fait désormais partie des lieux communs de la pensée et des sujets de conversation à la mode dans les dîners en ville.

Il a contribué à diffuser l'idée que les conflits à venir n'auront pas pour origine l'économie ou l'idéologie mais les lignes de fracture culturelle. Le sentiment d'appartenance à une civilisation a pris et continuera de prendre de plus en plus d'importance politique. Selon lui, la scène internationale sera dans une large mesure façonnée par les interactions de sept ou huit civilisations : occidentale, confucéenne, japonaise, islamique, hindouiste, slavo-orthodoxe, latino-américaine, africaine. Or, les concepts de la civilisation occidentale ont de moins en moins de validité et d'intelligibilité dans les autres civilisations. La conséquence politique directe réside dans des risques de guerre d'un genre nouveau où les enjeux sont non seulement liés au contrôle de population mais aussi de territoire en fonction des lignes d'influence des civilisations. Dans cette nouvelle configuration des rapports de force, l'islam occupe une place centrale. C'est ainsi que dans le New York Times, cité par Huntington, ont été recensés 48 endroits où se sont déroulés 59 conflits ethniques en 1993 et deux tiers de ces conflits ont eu lieu entre musulmans et non musulmans. L'islam serait donc 
caractérisé par des "frontières sanglantes». Le haut degré de militarisation des sociétés musulmanes est l'autre élément de preuve au cœur de l'argumentation: le potentiel militaire des pays «musulmans » serait deux fois supérieur à celui des pays " chrétiens ", de là à déduire qu'il y a une étroite corrélation entre islam et militarisme, le pas est vite franchi à l'aide de quelques statistiques d'armement. De même la propension à utiliser la violence dans les crises internationales serait un attribut de l'islam : selon de savants calculs du même ordre, sur les 142 crises dans lesquelles les « musulmans » ont été impliqués entre 1928 et 1979, 76 ont été résolues par la violence.

La première raison invoquée par $\mathrm{S}$. Huntington réside dans un atavisme musulman puisque l'islam serait une religion de l'épée glorifiant les vertus militaires. La vision d'un islam monolithique, lieu commun justifiant une approche pseudo-scientifique, conduit donc à un réductionnisme par lequel les conflits au Soudan, Liban, Bosnie, Azerbaïdjan relèvent d'enjeux religieux et similaires. Les critiques de cette vision essentialiste de l'islam, dénuée de toute connaissance du monde musulman et de son histoire, se sont élevées parmi les historiens et les spécialistes de l'islam, dès la parution de son article dans Foreign Affairs en $1993^{6}$.

Or la «variable» religieuse per se ne peut être surdéterminante. Il est d'ailleurs paradoxal de constater que son rôle si longtemps ignoré ou négligé dans les affaires internationales est maintenant exagéré et décontextualisé dans une perspective transhistorique qui en dit plus long sur la permanence d'un certain imaginaire que sur la réalité. Certes l'approche " huntigtonnienne " repose sur une prémisse qui ne peut être négligée : à savoir la place déterminante de l'identité et de la culture dans la configuration des relations internationales. Elle s'inscrit par ailleurs dans ce courant de recherche qui prend la mesure de la révolte contre l'Occident et alerte sur les perspectives ethno-centriques du projet des Lumières ${ }^{7}$. Mais l'approche substantialiste et non critique de la culture en général et de l'islam en particulier employée dans cette démonstration est quant à elle éminemment contestable.

Le retour de la culture et de l'identité dans l'approche internationaliste est lié à l'hétérogenéisation croissante de la scène internationale et à la fin des conflits exclusivement stato-centrés. En effet, la période de l'après-guerre froide oblige à revisiter les approches traditionnelles des relations internationales et à questionner les concepts habituels. La démarche la plus innovante réside vraisemblablement dans la mise en évidence du caractère historique de la politique internationale afin de prendre en compte le problème du changement autrement que sous le registre de la métaphore. Déconstruire les différentes narrations historiques serait alors une des tâches principales de l'internationaliste. Ceci inclut de remettre en cause la vision téléologique des Lumières telles qu'elle s'exprime dans la rhétorique de la fin de l'histoire comme de refuser la réification de concepts historiques dont par exemple celui de souveraineté Dans cette perspective, le recours aux concepts d'identité et de culture peut être pertinent à condition d'échapper à une vision essentialiste des deux termes, ce que ne fait justement pas Samuel Huntington. A cet égard, il est frappant de constater le retard entre l'approche sociologique et l'approche internationaliste de l'identité et de la culture.

Dans la sociologie de la culture ${ }^{9}, l^{\prime}$ accent est mis sur la fluidité et le caractère construit des identités et des processus culturels en opposition à une approche fixiste et naturaliste qui est encore dominante dans le champ de l'étude des relations internationales. Or, de telles orientations sociologiques ne peuvent être que judicieuses 
dans les recherches internationaliste car elles remettent en cause la vision dominante d'acteurs stables produisant et reproduisant un monde prévisible et incitent à prendre davantage en considération l'hétérogénéité et les contradictions des univers culturels et sociaux. La thèse du Clash des Civilisations représente justement une tentative non aboutie consistant, certes, à décentrer la politique internationale d'une approche exclusivement stato-centrée pour immédiatement recréer et légitimer un monde réifié d'agents culturels investis dans des conflits d'intérêts prédéfinis ${ }^{10}$. En d'autres termes toute tentative d'approche de la culture et des conflits culturels à l'échelle internationale est légitime mais ne peut s'élaborer sur une approche substantialiste de ces deux termes.

Or, à propos de l'islam, dans le débat public comme dans certains écrits à prétention scientifique, les "psychomachies » dominent, c'est-à-dire à la confrontation d'entités abstraites et rivales qui atteste que l'orientalisme latent a encore de beaux jours devant lui. Qui plus est, l'après-guerre froide favorise "l'illusion identitaire» selon le titre d'un ouvrage de Jean-Francois Bayart. Il est tentant de faire primer les facteurs culturels primordiaux comme explication des conflits et des troubles. C'est pourquoi, on ne peut que contester une vision de la culture islamique qui ne ferait de l'islam que le partenaire d'une "guerre des civilisations" que les grandes régions du monde seraient supposées se livrer. D'une certaine façon, un rejet bienveillant de cette caricature de l'islam - qui s'exprime par exemple par le "non à l'islamophobie » du dialogue "euro-islamique " - s'alimente aussi de cette vision car, en identifiant les conséquences "prévisibles » de ce « choc des cultures", il accrédite cette vision qui ne tient pas compte de la réalité des groupes humains, engagés dans une histoire et dans l'histoire du monde. Or, le comportement des acteurs dans les conflits nationaux, régionaux et mondiaux correspond à des logiques politiques variées qui sont incompréhensibles lorsqu'on refuse leur inscription dans le temps et dans l'espace.

Les fabrications et renversements d'alliance donnent une vision peu cohérente de la politique mondiale si l'on ne veut y voir que l'expression d'identitarismes basés sur telle religion, ethnie, nation ou culture. En revanche, une cohérence existe parmi les musulmans lorsqu'ils se mobilisent au moins par la parole dans les conflits les plus médiatisés. A cet égard, la Bosnie, la Tchéchénie, l'Algérie ou la Palestine représentent moins des «zones de fracture", que des «lieux-enjeux" où l'injustice faite aux populations victimes de l'histoire politique récente justifie la révolte et la radicalisation qui trouvent actuellement des possibilités d'expression dans les utilisations politiques de l'islam ${ }^{11}$. La mobilisation du monde musulman autour de questions comme la Bosnie, la Tchéchénie, ou la guerre du Kosovo, met en lumière comment, un certain nombre de valeurs liées à des registres culturels « non-occidentaux » représentent une alternative identitaire légitime. Cette mobilisation/identification est souvent déroutante pour l'observateur car ces valeurs ne sont pas toujours perceptibles dans les comportements quotidiens des acteurs, lesquels sont banalisés par leur insertion dans les réseaux mondiaux d'échanges commerciaux et culturels. Toutefois, c'est avec les langues et les cultures locales que l'on produit un sens politique qui dépend dans tous ses aspects des interférences internationales et des réseaux transfrontaliers qui se constituent.

La théorisation à l'échelle mondiale de concepts longtemps et exclusivement pensés nationalement, tels que citoyen, étranger, ethnicité, permet alors d'échapper à la vision réificatrice de la culture. Cela constitue également un moyen de sortir du schéma de fonctionnement de la pensée occidentale : celui du moi qui ne se reconnaît qu'à travers son autre, un autre qu'il choisit et façonne afin qu'il puisse jouer le rôle de 
structuration de son être. Il semble cependant que l'un des effets du 11 septembre est d'avoir ébranlé la croyance des puissances occidentales et particulièrement américaine à dominer la narration politique mondiale au nom de valeurs présentées comme universelles. Certes en Europe les discours de la puissance étaient déjà singulièrement émoussés par plusieurs siècles de guerre, de génocide et de décolonisation. Mais ils gardaient jusqu'à présent toute leur "fraîcheur" de l'autre côté de l'Atlantique. Assisterait-on depuis le 11 septembre à un "crépuscule des valeurs" de l'Occident désormais "trop sceptique pour être capable d'une lutte à mort?", s'interrogeaient les philosophes européens ${ }^{12}$. En fait, en ce qui concerne la politique américaine, rien n'est moins sûr car la victoire contre les Talibans en Afghanistan incite au triomphalisme et au sentiment invincible d'être dans son bon droit politique et même religieux si l'on se réfère au discours de George Bush du 30 janvier 2002 appelant à lutter contre l'axe du diable (axis evil).

Quelles sont les conséquences d'une telle situation sur les musulmans de l'intérieur tant européens qu'américains? De façon inattendue et surprenante, une fois passé «l'effet boomerang » des premières semaines, la dissociation entre ennemi politique et musulman de l'intérieur se dessine. Cette différenciation vient valider l'approche de la mondialisation culturelle qui met l'accent sur l'hétérogéneité croissante de la scène internationale ${ }^{13}$, c'est-à-dire sur les processus d'hybridation, de mélange mais aussi de différentiation, de distinction et de résistance aux cultures occidentales dominantes. Prenant à contre-pied la perception essentialiste de l'islam, l'hétérogoneisation culturelle permet pour la première fois d'introduire une différence entre musulmans en fonction du contexte dans lequel ils vivent. Cette acceptation bon gré malgré de la différentiation a été grandement favorisée par la mobilisation depuis plusieurs années des musulmans européens et américains en faveur de la distinction entre " eux » (du monde musulman) et "nous", musulmans du monde démocratique. En d'autres termes, l'un des effets de la mondialisation est non seulement de rendre possible l'émergence des musulmans de l'Occident mais aussi pour la première fois d'introduire une fissure dans le disours unificateur sur l'islam.

L'ennemi intérieur : les ambivalences de l'après 11 septembre

Certes «l'effet ben Laden" a dans un premier temps consisté à appliquer la vision de l'ennemi aux musulmans de l'intérieur, aux Etats-Unis et en Europe, les transformant en boucs émissaires, selon un processus bien connu. Des centaines d'attaques verbales et physiques, dont certaines meurtrières contre des personnes au faciès arabe ou musulman ont été recensées aux Etats-Unis. En Europe, les agressions physiques recensées ont été moins nombreuses mais des insultes et des agressions verbales ont été raportées dans la plupart des pays européens particulièrement en Grande-Bretagne, en Allemagne et aux Pays-Bas. Les actes de discrimination dans les transports et les bâtiments publics se sont également multipliés, certains spectaculaires comme par exemple le personnel naviguant d'une compagnie américaine refusant de décoller avec des passagers musulmans à son bord.

Toutefois l'attitude des populations et des gouvernements s'est en fait révélée plus ambivalente à l'égard des musulmans de l'intérieur que ces premières réactions à chaud peuvent le laisser croire, associant répression et discrimination à une volonté de compréhension et de dialogue, y compris aux Etats-Unis. La plupart des sondages en Europe montrent que depuis le 11 septembre, les populations n'ont pas diamétralement changé d'avis concernant les musulmans. L'image de l'islam était globalement négative en Europe et aux Etats-Unis et elle l'est restée. Par exemple en France, la population 
non musulmane continue d'associer islam avec fanatisme avec une belle régularité depuis 1994. De même aux Etats-Unis, les récents sondages démontrent cette association entre islam et fanatisme ${ }^{14}$. Il y aurait toutefois un effet positif lié à l'effet ben Laden et à l'intérêt accru pour la culture et la civilisation islamiques qui s'est traduit partout par une hausse exponentielle d'achats de livres, de discours publics et médiatiques. Aux Etats-Unis, même après le 11 septembre, l'islam attire toujours des prosélytes $^{15}$. En même temps dans certains pays comme l'Allemagne, les Pays-Bas, le Portugal ou la Suède, la suspicion envers les musulmans s'est aggravée, ceux-ci étant dans l'obligation de justifier en permanence leur désapprobation du terrorisme. L'attitude des media a aussi contribué à renforcer les stéréotypes et préjudices en matière d'islam, particulièrement aux Pays-Bas, en Grèce, en Irlande, en Italie, etc. ${ }^{16}$ Du côté des gouvernements, un des autres aspects positifs réside dans un discours explicite tendant à distinguer entre l'événement international et la condition de l'islam dans le pays concerné. Les initiatives pour créer des liens et des espaces de dialogue avec les musulmans ainsi qu'avec d'autres groupes religieux ont été lancées au Danemark, en Allemagne, en Italie ou aux Pays-Bas, tandis que dans des pays où le dialogue existait déjà comme en France, en Grande Bretagne ou en Belgique, il s'en est trouvé renforcé. Aux Etats-Unis, l'après 11 septembre a eu également comme conséquence de multiplier les espaces de dialogue entre leaders musulmans et représentants des pouvoirs publics tant au niveau fédéral qu'à celui des différents Etats.

<!--SPIP--> En même temps, il est clair que cette attaque terroriste a durci le discours sur l'immigration (Autriche, Danemark, Allemagne, Grèce, Italie et Portugal) et sur la sécurité. La loi anti-terroriste ratifiée par George Bush le 26 octobre 2001 donnant des pouvoirs étendus en matière de contrôle des citoyens - y compris de leur famille et $\mathrm{y}$ compris de ceux qui ne sont pas citoyens américains - a été suivie par des initiatives comparables en Europe. En Grande-Bretagne, une loi sur l'anti-terrorisme, le crime et la sécurité est passée le 14 décembre 2001 et a soulevé un vrai débat sur la restriction des libertés publiques car elle aussi donne des pouvoirs accrus à la police en matière de recherche d'informations et de contrôle des citoyens. En Allemagne, deux lois ont été votées, l'une le 8 décembre, la seconde le 20 décembre 2001. Elles accroissent non seulement les moyens financiers des forces de sécurité mais aussi leurs pouvoirs d'investigation, prévoient des agents de sécurité armés sur les avions allemands et une révision de la loi accordant des privilèges aux organisations religieuses en tant que corporation de droit public. Le débat sur la sécurité, s'est trouvé subverti par les événements du 11 septembre et la prise en compte du contre-terrorisme comme l'atteste la loi française promulguée le 15 novembre 2001 sur la sécurité au quotidien et dans laquelle a été introduit toute une série de rubriques sur la lutte anti-terrorisme lors des débats intervenus à l'Assemblée nationale après le 11 septembre, amalgamant ainsi sécurité intérieure, délinquance et terrorisme, ce qui contribue à intensifier l'ostracisme des jeunes des banlieues. En particulier, deux des mesures de la loi - celle concernant la tranquillité dans les halls des immeubles collectifs, et celle faisant encourir une peine maximale de six mois de prison aux fraudeurs « habituels » dans les transports en commun - n'ont semble-t-il aucune relation et n'auront d'ailleurs aucun impact sur la grande délinquance et le terrorisme.

Cependant en France comme ailleurs en Europe, l'ambivalence règne en matière de perception de l'islam et des musulmans. Certes, du point de vue des libertés publiques, le débat français peut sembler faire piètre figure comparé notamment à celui du monde anglo-saxon. Le vote de la loi sur la sécurité quotidienne n'a pas soulevé de véritable 
débat public à l'exception de l'émoi de quelques intellectuels ${ }^{17}$. En même temps, du point de vue de la perception de l'islam comme religion dans la société civile, des signes d'apaisement se font jour qui sont loin d'avoir été affaiblis par le 11 septembre. Une nouvelle forme française d'exception serait-elle en train d'émerger, faite de plus de tolérance par rapport au fait musulman? Ce paradoxe se retrouve également aux EtatsUnis où les américains déclarent avoir une bonne image des musulmans américains et une image négative de l'islam ${ }^{18}$ et où l'une des conséquences du 11 septembre est la mise en lumière et la soudaine centralité dans le débat public de la minorité musulmane américaine.

Vers une atténuation du syndrome post-colonial français?

Pour comprendre cette évolution française, il faut se rappeler que le "prisme néocolonial » a été (et reste encore) la principale grille de lecture de l'islam tant sur le plan domestique qu'international. La guerre d'Algérie, plus politiquement que militairement perdue, a ouvert une plaie dans l'identité nationale française, avivée par la sédentarisation dans l'hexagone, des anciens colonisés. Comme toute crise d'identité, elle a trait à la mémoire, au maintien de soi à travers le temps. Dans cette mémoire, les menaces réelles ou imaginaires liées à la confrontation avec l'autre musulman, tiennent une place centrale. La place de la violence, dans la construction des identités collectives, ajoute un élément supplémentaire de vulnérabilité.

De 1962 jusqu'à très récemment, un mélange de mutisme, d'oubli et de ressentiment a prévalu. Depuis une dizaine d'années toutefois, il est difficile de considérer cette séquence historique comme absente de nos mémoires. En effet, témoignages, livres, films, expositions se sont multipliés en l'espace de quelques années : entre 1991 et 1992, pas moins de six documentaires ont traité de cet épisode à la télévision ou au cinéma : "Les années algériennes, la guerre sans nom ", "Les Lettres de soldats d'Algérie ", "Mémoire d'Algérie », «Guerre d'Algérie », etc. Mais il s'agit d'un moment de la sortie $\mathrm{du}$ silence et pas encore de la commémoration. Cette fin de l'amnésie prendra vraisemblablement encore du temps dans la mesure où cette guerre n'a pas été insérée dans la mémoire commune à part quelques "fragments " (harkis, rapatriés) et fait encore largement partie du domaine privé. Face à cette béance, le geste en 1996, renouvelé à maintes reprises, du président Jacques Chirac d'associer aux commémorations du 11 novembre les anciens combattants d'Algérie est tout à la fois symboliquement significatif et d'une portée très limitée. La polémique qui fait toujours rage sur la reconnaissance de la journée du 17 octobre 1961 est significative de cette douloureuse sortie du mutisme ${ }^{19}$.

C'est ainsi que, depuis presque trente ans maintenant, se produit un étrange balancement de l'opinion publique entre d'un côté, l'axe de la volition et de la mythologie de l'humanisme républicain (France terre d'accueil) et de l'autre celui du repli, de la méfiance et de la peur de la perte de substance. Il faut à cet égard rappeler que bien avant le Front national, la crise algérienne et l'élaboration d'un étranger-type sont à l'origine du retour de l'extrême droite dans le paysage politique : qu'il s'agisse du Rassemblement National de Jean-Louis Tixier-Vignancour et de l'Union de défense des commerçants et artisans de Pierre Poujade qui en mai 1957 s'alliera pour la défense de l'Algérie française avec Henri Dorgères.

La rhétorique xénophobe et différentialiste progresse à la faveur de la crise d'identité française avec, comme ligne d'horizon, le spectre d'une "guerre des cultures». Au cœur de cette confrontation, il y a les derniers arrivés venus du Maghreb et d'Afrique. 
Anciens colonisés et qui plus est musulmans, ils ne sont pas des immigrés et a fortiori des citoyens comme les autres. L'intégration républicaine trouve ici ses limites dans une perception et une prise en compte différenciée des personnes en fonction de l'origine dont le Front National n'a pas l'apanage, mais qu'il sait exploiter à son avantage.

Le régime de Vichy puis la décolonisation ont ainsi contribué à ébranler de manière irréversible, la croyance en l'universel de la République et en ses symboles intégrateurs. C'est désormais l'impératif de "naturalisation » des anciens «dominés » qui vient affaiblir l'efficacité, amoindrie, du creuset républicain. Par ailleurs, pour les groupes issus de l'immigration maghrébine, l'accès à la citoyenneté universelle est faussé par leur condition post-coloniale. Lorsque ces anciens "sujets" sont devenus des immigrés et leurs enfants des citoyens français, ces derniers n'ont jamais été acceptés pleinement comme tels. Toutes les étapes du débat sur l'immigration depuis plus de vingt ans maintenant attestent de cette incapacité à faire une place dans l'égalité et la fraternité à cet « autre $»^{20}$.

Cette spécificité française réactivée dans le débat contemporain sur l'immigration maghrébine a été amplifiée pendant longtemps par la vision internationale de l'islam décrite précédemment. Ces musulmans de l'intérieur portent en eux trois différences monstrueuses : la religion, l'ethnie et la pauvreté. Leurs actions et revendications sont le plus souvent appréciées à l'aune des troubles internationaux, ce qui légitime et renforce leur marginalité dans la communauté nationale. La suspicion sur le loyalisme de ces nouveaux citoyens s'est particulièrement illustrée en France au moment de la deuxième guerre du Golfe contre l'Irak et surtout avec les prolongations de la crise algérienne dans l'hexagone depuis 1994 et la mise en place du premier plan Vigipirate. C'est ainsi que durant cette période, le fait social et religieux islamique a été traité comme un fait sécuritaire.

L'islam français comme enjeu sécuritaire

Lorsque les immigrés ont construit des salles de prière dans les cités HLM au début des années 1980, les esprits les plus éclairés y compris dans le monde scientifique, n'ont pas hésité à parler de « retour de l'islam » alors que ces personnes n'étaient pas devenues plus pratiquantes mais avaient simplement changé leur attitude vis-à-vis de la société française, revendiquant désormais des lieux de culte. De manière plus significative encore, l'actualité internationale a été sollicitée comme facteur explicatif principal de cette émergence publique de l'islam dans les banlieues. Ce nouveau phénomène social a été à son origine interprété comme la conséquence de manipulations extérieures et donc comme un facteur de déstabilisation politique car il se situe au moment où l'islam surgit sur la scène internationale et devient un élément de la contestation politique dans le monde arabo-islamique. C'est donc à l'aune de la révolution islamique en Iran et de l'essor des mouvements islamistes en Egypte ou au Maghreb que va être appréciée et pis encore analysée la demande d'islam dans les entreprises ou les banlieues selon la plus belle logique du complot.

Ce plaquage de la situation internationale sur le contexte français a contribué à une lecture politique des manifestations islamiques françaises, sans cesse réactivée par des troubles extérieurs. Par exemple, associer systématiquement la crise politique algérienne à toute forme de revendication islamique dans l'hexagone revenait à occulter les dynamiques sociales et culturelles innovantes propres aux musulmans transplantés ${ }^{21}$. 
Cette externalisation de l'islam est également très significative dans la perception politique de l'islam français. Jusqu'au début des années 1980, les gouvernants français avaient pris l'habitude de considérer les dirigeants des principaux pays maghrébins et notamment l'Algérie comme leurs principaux interlocuteurs pour toutes les questions relatives à l'islam. Dans cette perspective, la Mosquée de Paris a constitué un élément décisif dans les relations diplomatiques entre la France et l'Algérie. Legs de la période coloniale, la controverse autour de ce lieu recèle bien la permanence du prisme « algérien » appliqué à l'islam de France.

Cette vision sécuritaire a été amplifiée par la crise politique algérienne des années 1990 qui a favorisé une réactivation des schémas de pensée habituels. A cet égard, la collusion entre ennemi intérieur et ennemi extérieur s'est parfaitement illustrée lors de l'instauration du dispositif Vigipirate à la suite des attentats dans le métro parisien en 1995. Le corollaire de ce dispositif sécuritaire a été la suspicion généralisée sur toute forme de religiosité islamique en particulier lorsqu'elle émane de la jeunesse des banlieues. Le syndrome post-colonial se doublait donc d'un syndrome Khaled Kelkal, du nom du jeune lyonnais d'origine algérienne impliqué dans les attentats du métro parisien et abattu par la police au cours d'une course poursuite.

Pour bon nombre de jeunes pratiquants, la relation à l'environnement est alors doublement difficile. Tout d'abord en tant que musulmans, ils subissent la méfiance ou le rejet au quotidien qui se doublent, dans le cas où ils sont militants associatifs, d'une relégation institutionnelle. S'afficher comme musulman dans le contexte français revient bien souvent à expérimenter certaines formes d'indignité culturelle. Cette expérience de l'indignité liée à l'identité musulmane est avivée par le syndrome postcolonial, qui dans leur cas, les oblige à penser que c'est non seulement la religion mais aussi l'origine arabe qui est ainsi mise hors-la-loi dans la société française. A cela s'ajoute la diabolisation permanente de l'islam liée aux enjeux géopolitiques, de l'Afghanistan à la Bosnie, et surtout l'Algérie, qui ne fait qu'attiser le sentiment d'injustice et de relégation.

Ce sentiment d'indignité à titre personnel se trouve en quelque sorte renforcé par la relégation institutionnelle que subissent certaines associations dès lors qu'elles sont dirigées par des "musulmans». Le processus décrit est toujours le même: des subventions peuvent être données au moment de la création mais lorsque le caractère islamique des membres est avéré, l'aide institutionnelle s'arrête. Cette relégation institutionnelle s'accompagne d'une difficulté de dialogue voire d'une absence de dialogue avec les représentants du travail social ou d'autres formes d'intermédiaires dans le quartier ou la cité. La crise algérienne et la mise en place du dispositif Vigipirate ont renforcé cet engrenage de la suspicion. A la relégation institutionnelle s'ajoutent désormais des tracasseries administratives et policières plus fréquentes qui renforcent leur sentiment de fragilité et font naitre également la peur.

Les différents témoignages recueillis à cette période mettent en lumière deux logiques antagoniques: d'un côté, la perception par les représentants institutionnels de ces nouveaux militants comme " des maîtres du double langage » qui montrent une image acceptable mais dont les buts seraient in fine moins honorables, de l'autre, des acteurs pris dans la contradiction du souci de reconnaissance et de préservation de leur indépendance. De plus, l'islamophobie permanente qu'ils ont à affronter dans leur vie quotidienne comme dans le discours politique et intellectuel dominant, n'arrange rien. 
Si le sentiment d'indignité culturelle persiste, la phase d'hostilité ouverte s'est émoussée du moins dans la relation aux institutions. Les résistances à considérer l'islam comme composante à part entière du paysage religieux et culturel français se sont sans conteste atténuées au cours de la décennie 1990 en particulier au niveau local. La destruction d'une salle de prière par un bulldozer comme à CharvieuChavagneux en 1989 ou le référendum organisé à Libercourt en 1991 par le maire pour demander l'avis des citoyens sur le projet de mosquée en cours, sont autant d'épisodes noirs qui font partie du passé de la relation entre l'islam et la République. Il ne viendrait plus désormais à l'esprit d'aucun édile local de dénier, au moins officiellement, le droit à l'existence d'une mosquée dans sa commune et presque partout des relations se sont établies entre représentants associatifs du monde musulman et pouvoirs publics. Cette concertation locale a eu comme conséquence de légitimer l'islam comme fait social et culturel dans la vie publique française. Un dialogue presque permanent est en place entre édiles locaux et responsables associatifs islamiques. Ce dialogue est en train d'inclure la nouvelle génération de musulmans éduqués dans le système français et membres des classes moyennes ${ }^{22}$. Les derniers bastions de résistance à la construction de mosquées sont en train de tomber comme à Toulouse, ou encore dans le 19e arrondissement de Paris qui jusqu'en 2001 refusait le permis de construire au projet de la mosquée Adda'wa.

$\mathrm{Au}$ niveau national également le dialogue s'est institutionnalisé. A partir de 1988, la perception de l'islam se modifie. La primauté accordée à la mosquée de Paris a cédé la place à la prise en compte de la diversité religieuse et ethnique de l'islam français. Pierre Joxe, alors Ministre de l'Intérieur réunissait au mois de novembre 1989 une commission de six « sages » musulmans, élargie en mars 1990 sous le nom de «Conseil de Réflexion sur l'Islam en France » (Corif) L'ambition recherchée était l'unification des populations musulmanes afin que le gouvernement puisse bénéficier d'un interlocuteur unique et "avisé ». Si le Corif a échoué, le principe d'une consultation élargie incluant toutes les tendances ethniques et religieuses de l'islam français est quant à lui resté à l'ordre du jour. La tentative la plus récente pour sortir de l'impasse est la consultation de l'ensemble des leaders islamiques lancée par Jean-Pierre Chevènement, ministre de l'Intérieur, en octobre 1999 et qui a abouti à la signature par tous le 28 janvier 2000, d'un document consacrant une fois de plus la compatibilité de l'islam et de la République. Reprise et amplifiée par Daniel Vaillant, le successeur de Jean-Pierre Chevènement à la place Beauveau à partir de septembre 2000, un pas supplémentaire est franchi le 3 juillet 2001 lorsque l'ensemble des associations et personnalités islamiques consultées adoptent un accord-cadre sur l'organisation future du culte musulman qui prendra la forme d'une association de la loi de 1901 qui devra conduire à l'élection de conseillers régionaux et d'une instance centrale qui prendra le nom de "Conseil français du Culte musulman ». Le 11 septembre n'a pas remis en cause cette dynamique bien au contraire, en dépit de certaines critiques émises par certaines figures musulmanes de l'intérieur même de la consultation ${ }^{23}$.

Il semblerait donc que pour la première fois dans l'histoire de l'islam français des vingt dernières années, la logique internationale n'affecte pas la dynamique de reconnaissance de l'islam comme fait social. L'enracinement de l'islam dans les espaces locaux en est certainement la raison. Il se produit une dissociation étonnante et paradoxale entre le voisin musulman, connu si ce n'est reconnu et l'islam, terme encore inconnu et souvent porteur des imageries les plus négatives ${ }^{24}$. 


\section{NOTES}

1. . A propos de cette orientalisation de l'islam, voir Turner, B.S., Orientalism, Postmodernism and Globalism, Londres, Routledge, 1994.

2. A propos de la composition/activation de ces images, nous nous permettons de référer à Cesari, J., Faut-il avoir peur de l'islam ?, Paris, Presses de Sciences Po, 1997.

3. . Paris, Seuil, 1980.

4. . Renan, E., L'islamisme et la science, Oeuvres complètes, Tome I, ed. Henriette Psichari, Paris, Calmann Levy, 1947-61, p. 956.

5. . Cette section est issue de Cesari, J., Faut-il avoir peur de l'Islam ?, op. cit.

6. . Voir Mottahedeh, R., « The Clash of Civilizations : An Islamicist's critique », Harvard Middle Eastern and Islamic Review, 2 (1), Cambridge, Cambridge University Press, 1995, pp. 1-26. Voir également « Troubler et Inquiéter. Les discours du désordre international, Cultures \& Conflits, n¹9/20, Paris, L'Harmattan, automne-hiver 1995. 7. . Voir Bozeman, A., « The International Order in a Multicultural World. The Expansion of International Society » in Hedley B. and Watson A., The Expansion of International Society, Oxford, Clarendon, 1984.

8. . Lapid,Y. et Kratochwil, F., The Return of Culture and Identity in IR Theory, Londres, Lynne Rienner Publishers, Boulder, 1996, p. 216.

9. . Featherstone, M., Global Culture : Nationalism, Globalization and Modernity, Londres, Sage, 1990 ; Hannerz, U., Transnational Connections : Culture, People, Places, Londres, Routledge, 1996 ; Robertson, R., Globalization : Social Theory and Global Culture, Londres, Sage, 1992.

10. . Voir Rubenstein, R.E. and Crocker, J, « Challenging Huntigton », Foreign Policy, pp. 113-128.

11. . Pour sortir de ces impasses de l'explication, il convient, nous semble-t-il, d'essayer de dégager les logiques politiques qui interviennent dans les utilisations de l'islam comme référent explicatif. Au-delà du comportement des musulmans eux-mêmes en tant qu'individus, la crise des idéologies et des territoires, dramatisée par les illusions de la mondialisation, dessine trois types de logiques dans les stratégies politiques qui ont des effets sur les relations internationales.

1) Certaines stratégies sont dictées par des intérêts nationaux ou locaux (ethniques, de clans, etc.). Si leurs acteurs utilisent volontiers le vocabulaire de l'islam, leurs visées ont peu à voir avec la réalité culturelle de l'islam. On peut expliquer ainsi l'absence de soutien de l'Iran, en 1992, aux chi'ites irakiens attaqués par le régime de Saddam Hussein ; ou la préférence donnée par les mouvements islamistes palestiniens et libanais à la reconquête du territoire national, sur l'application de la loi islamique dans les territoires qu'ils contrôlent; ou encore les alliances réalisées par le Pakistan de Benazir Bhutto avec les islamistes de Hekmatyar, puis avec ceux du mouvement des talibans.

2) Ces stratégies nationales s'inscrivent ensuite dans des impératifs régionaux particuliers. Par exemple, rien ne semblait annoncer une concertation aussi rapide de la Turquie, de la Syrie, de l'Irak et de l'Iran, réalisée en août 1996 : si ce n'est les possibilités de faire échec à la politique israélo-américaine en Palestine, ouvertes par l'accession au pouvoir du parti islamiste Refah en Turquie. On peut tout aussi bien attribuer à des divergences d'analyse des intérêts économiques liés à la région, la 
réticence des Européens à suivre les Américains dans le système de sanctions contre l'Iran. Sur un autre plan, le monde occidental, soucieux de ménager ses bases pétrolières est resté longtemps indifférent à l'application de la loi islamique en Arabie saoudite, sur un mode contesté par une opposition interne qui réclame moins d'arbitraire dans la gestion politique ; ou encore la Russie choisissant de montrer sa force en Tchétchénie, pays par lequel transite le pétrole de la Caspienne.

3) Mais ces logiques, nationales et régionales semblent transcendées par celles découlant du projet de mondialisation ou de ce qui est perçu comme tel. La seconde guerre du Golfe (1990-91) a produit des alliances inattendues avec l'Irak : soutien des islamistes algériens comme de nombreux mouvements révolutionnaires, islamistes ou non (chez les Palestiniens en particulier). De façon chronique, la question palestinienne constitue un lieu de conflit potentiel-type, puisque l'on peut poser que ce conflit ne peut recevoir un début de solution si l'injustice faite aux Palestiniens, et à travers eux, aux musulmans, n'est pas résolue. Voir Botiveau, B. et Cesari, J., Géopolitique des Islams, Paris, Economica, 1997.

12. . Propos du philosophe Gianni Vattimo recueillis dans Le Monde, 18 octobre 2001. 13. . Voir Bryan S.Turner, Orientalism, Postmodernism and Globalism, Londres, Routledge, 1994 ; Appadurai, Arjun, « Disjuncture and Difference in the Global Cultural Economy » in Featherstone, M. (ed), Global Culture, Nationalism, Globalization and Modernity, Londres, Sage Publication, 1990, pp. 295-311 ou encore Hannerz, Ulf, Cultural Complexity : studies in the Social Organization of Meaning, New York, Columbia University Press, 1992.

14. . Sondage IFOP du 5 octobre 2001 réalisé auprès d'un échantillon de 940 non musulmans et 548 personnes ayant déclaré appartenir à une famille musulmane. Sondage New Reuters/ Zogby poll, 17 septembre 2001, réalisé auprès d'un échantillon de 1018 personnes.

15. . Voir « Islam attracts converts by the thousands, drawn before and after the attacks », The New York Times, 22 octobre 2001.

16. . European Monitoring Center on Racism and Xenophobia, Anti-Islamic reactions in the EU after the terrorist acts against the USA, Vienna, 29 novembre 2001.

17. . Voir pétition parue dans Le Monde du 12 décembre 2001.

18. . Voir sondage Reuters/Zogby du 17 septembre déjà cité. Les Américains savent aussi depuis l'attentat d'Oklahoma City en 1995 que l'ennemi, en particulier dans la mouvance de l'extrême-droite, peut être produit du cœur même de la société américaine.

19. . Le 17 octobre 1961, une manifestation organisée par des Algériens, en soutien au FLN, eut lieu à Paris et fut violemment réprimée par la police. Elle se solda par des arrestations massives et des assassinats dont le chiffre des victimes n'a pas encore été formellement établi.

20. . Comment expliquer par exemple l'usage immodéré de la formule « deuxième génération » pour désigner ceux qui, selon le droit du sol ne devraient être que de jeunes français ? Faut-il rappeler qu'à aucun moment dans l'histoire des migrations, pourtant fort nombreuses dans ce pays, les enfants d'immigrés polonais, italiens ou portugais n'ont été qualifiés de manière aussi explicite de « deuxième génération »? De même, comment comprendre cette distinction entre français de souche et français d'origine étrangère qui s'est également largement répandue et qui en vertu même de la logique républicaine devrait être bannie ? Pourquoi le critère ethnique entre-t-il implicitement en ligne de compte dans le traitement social des banlieues et 
d'amalgames en glissements de sens arrive-t-on à l'équation banlieues=immigration=arabes qui sous-tend les politiques les plus généreuses en apparence et conduit à une utilisation très pernicieuse du terme intégration, réservé exclusivement aux personnes d'origine immigrée ? Il y a en effet l'idée que ces exclus sont dans leur plus grand nombre issus des dernières vagues migratoires et donc d'origine maghrébine. Or, cette perception est loin de rendre compte de la réalité sociale qui est très hétérogène et " métissée ".

21. . Voir notamment Cesari, J., Musulmans et Républicains, L'islam, les jeunes et la France, Bruxelles, Complexe, 1998.

22. . Le sondage IFOP du 5 octobre 2001 révèle cet effet de génération en mettant en lumière tout un groupe de personnes, nées en France, éduquées et pratiquantes.

23. . Voir Vaillant, D., « Les enjeux de la consultation des musulmans de France », Le Monde, 29 décembre 2001.

24. . Le sondage IFOP du 5 octobre met en lumière ce paradoxe puisque la majorité de l'échantillon non musulman se déclare soit indifférente soit en faveur de la construction de mosquées tout en associant islam et fanatisme.

\section{RÉSUMÉS}

Jocelyne Césari analyse les enjeux de citoyenneté pour les pratiquants de l'Islam, pour les minorités et pour les migrants et montre comment le profilage et la dynamique de suspicion qui se mettent en place dans l'après 11 septembre peuvent remettre en cause les valeurs d'ouverture, d'intégration qui font partie de l'image même des Etats-Unis d'Amérique. Dans cet article, l'auteur montre comment dans l'après 11 septembre se sont renforcés la vision sécuritaire et les conditions pour une dissociation entre le musulman de l'intérieur et l'ennemi politique.

Jocelyne Cesari analyses the stakes of citizenship for practising Muslims, minorities and migrants and shows how the post September 11th profiling and suspicion dynamic can put into question the values of openness and integration that characterise the United States of America. The author shows in this article how the vision of security and the condition for dissociation between the Muslim from the inside and the political enemy have become stronger.

INDEX

Index géographique : Etats-Unis

Index chronologique : 2001 post 11 septembre

Mots-clés : religion, menace, exclusion 